\title{
Pulmonary Vein Isolation for the Treatment of Drug-Refractory Atrial Fibrillation in Adults with Congenital Heart Disease
}

\author{
Femi Philip, MD, ${ }^{*}$ Kamran I. Muhammad, MD, ${ }^{*}$ Shikar Agarwal, MD, ${ }^{*}$ Andrea Natale, $M D,{ }^{\dagger}$ and \\ Richard A. Krasuski, MD* \\ *Department of Cardiovascular Medicine, Cleveland Clinic Foundation, Cleveland, Ohio; 'Texas Cardiac Arrhythmia \\ Institute, St David Medical Center, Austin, Tex, USA
}

\section{A B S T R A C T}

Background. Atrial fibrillation (AF) is a common arrhythmia in adults with congenital heart disease (CHD). Long-term antiarrhythmic therapy (AAT) in these patients has significant shortcomings. The safety and efficacy of pulmonary vein antrum isolation (PVAI) for the treatment of AF in CHD is presently unknown.

Hypothesis. We hypothesized that PVAI for AF in patients with CHD is effective and safe.

Methods. We reviewed a prospective cohort of 4315 patients (age $\geq 18$ ) undergoing PVAI for drug refractory AF at a single institution and identified 36 consecutive patients with CHD (single ventricle physiology, tetralogy of Fallot, coarctation of the aorta, ventricular septal defects, atrial septal defects (ASD) and cardiomyopathy resulting from anomalous origin of the left main coronary from the pulmonary artery). A second cohort of 355 consecutive patients with noncongenital structural heart disease (NSHD) (coronary artery disease, valvular heart disease, ejection fraction $<50 \%$, or prior noncongenital cardiac surgery) undergoing PVAI during the same time period was used as a control. Success was defined as freedom from AF starting two months after PVAI in the absence AAT until the end of follow-up. Partial success was defined as freedom from AF in the presence of AAT until the end of follow-up. Combined success was defined as the sum of success and partial success. We compared the outcomes with the use of propensity-score matching in the overall cohort.

Results. Patients with NSHD were older and had higher prevalence of hypertension $(P<.01)$, diabetes $(P<.01)$ and hyperlipidemia $(P<.01)$. The most common CHD lesion was ASD $(61 \%)$ and the most common NSHD lesion was valvular heart disease (57\%). After one PVAI, success was achieved in $42 \%$ and $53 \%$ at 300 days in the CHD and NSHD groups respectively. Four-year success was achieved in $27 \%$ and $36 \%$ in the CHD and NSHD groups, respectively. There were no significant differences in the success rates between patients groups $(P=.46)$, nor were there any differences in left atrial size or changes in ejection fraction after one or two PVAI in the respective groups. Complication rates between the CHD and NSHD groups were similar $(15 \%$ vs. $11 \%, P=.42)$ except for a higher risk of vascular site complications in patients with CHD ( $8 \%$ vs. $1 \%, P<.05)$.

Conclusion. PVAI is an attractive treatment modality in drug refractory AF in CHD, with combined success rates in excess of $60 \%$. The maintenance of sinus rhythm after PVAI in CHD appears similar to that of NSHD and warrants prospective validation.

Key Words. Adult Congenital Heart Disease; Atrial Fibrillation; Pulmonary Vein Isolation; Antiarrhythmic Therapy; Structural Heart Disease

\section{Introduction}

$\mathrm{T}$ he existence of a substantial population of adults born with congenital heart disease (CHD) is a new phenomenon. ${ }^{1}$ Despite ever improving survival, this population presents a novel set of challenges related both to their unique

Congenit Heart Dis. 2012;7:392-399 anatomy and to the sequelae of prior percutanous and surgical management. Among the more difficult problems that this group faces is an increased incidence of arrhythmias. Arrhythmias arise due to unique myocardial substrate created by extensive suture lines and long-standing, abnormal pressure-volume relationships. 
Atrial fibrillation (AF) is a very common arrhythmia in CHD and has well-known associations with advancing age and the presence of structural heart disease. ${ }^{2}$ Several prior clinical trials have examined the various therapeutic strategies in AF. In particular, the AF Follow-up Investigation of Rhythm Management (AFFIRM) ${ }^{3}$ Rate Control Versus Electric Cardioversion for AF (RACE), ${ }^{4}$ and Strategies for Treatment of AF (STAF) ${ }^{5}$ compared treatment strategies of rate control vs. rhythm control using antiarrhythmic drugs. Using an intention-to-treat analysis, these trials concluded that there was no mortality difference between the rate control and rhythm control strategies, implying no advantage in maintaining sinus rhythm. These trials, however, were limited by an inability to maintain sinus rhythm in either the rhythm control or the rate control arms: AFFIRM $(62.2 \%, 34 \%)$, RACE $(38.7 \%, 10 \%)$, and STAF (38\%, 9\%) respectively. Additionally, many patients in the rate-controlled arm were in sinus rhythm at the end of the study periods $(35 \%$ AFFIRM, $10 \%$ RACE and 10\% STAF respectively). These data demonstrate the ineffectiveness of maintaining sinus rhythm using antiarrhythmic drugs. A reduction in mortality with the maintenance of sinus rhythm was demonstrated in the Danish Investigations of Arrhythmia and Mortality on Dofetilide trail (DIAMOND) ${ }^{6}$ and Veterans Affairs Congestive Heart Failure Trial of Antiarrhythmic Therapy trail (CHF-STAT), ${ }^{7}$ although treatment with antiarrhythmics has generally been associated with increased mortality. A re-analysis of the AFFRIM trial has shown that the maintenance of sinus rhythm was associated with improved survival, and rhythm control with AAT did not have a high success rate therefore offsetting the mortality benefit in the rhythm control arm. ${ }^{8}$

These observations make a curative approach to the treatment of AF particularly attractive. One such approach is the use of pulmonary vein antrum isolation (PVAI) to electrically disconnect the pulmonary veins from the rest of the atrium. PVAI has a success rate of about $80-90 \%$ in selected patient populations. ${ }^{9}$ Additionally; PVAI may serve to improve left ventricular function and decrease chamber size in patients with congestive heart failure. ${ }^{10}$

It is important to note that none of the previously mentioned trials evaluated the use of PVAI in patients with congenital heart disease. Because of the more complex electrical substrate, it is uncertain whether this technique could also be successful in this patient population. We attempted to answer this question by examining a prospectively collected database of patients with CHD and drug refractory AF who underwent PVAI. We compared the success and complications rates of in patients with CHD to those with noncongenital structural heart disease (NSHD), a population in whom PVAI is commonly performed.

\section{Methods}

\section{Study Design}

We reviewed a retrospective study using a prospective cohort of 4315 patients undergoing PVAI for drug refractory $\mathrm{AF}$ and identified consecutive patients aged $\geq 18$ years with CHD who were to have their first PVAI ablation attempt between January 2004 until December 2010. All patients with CHD who had had an AF ablation were included, and this group consisted of patients with repaired and unrepaired atrial septal defects, repaired ventricular septal defects, tetralogy of Fallot, coarctation of the aorta, single ventricle physiology, transposition of the great vessels, Ebstein's anomaly, and congenital cardiomyopathy resulting from anomalous origin of the left main coronary artery from the pulmonary artery. A similar cohort of patients who had PVAI with noncongenital structural heart disease (NSHD) (defined as patients age $>18$ with coronary artery disease, valvular heart disease, or ejection fraction less than $50 \%$ and who were due to have their first PVAI) was used as a control group. The study was approved by the institutional ethics and review board of the Cleveland Clinic Foundation. All patients were initially seen by an electrophysiologist in the outpatient setting and determined to be appropriate for PVAI, and patients who had had prior PVAI attempts were excluded. Blood work included electrolytes, complete blood count, ultrasensitive C-reactive protein (CRP), brain natriuretic peptide (BNP), and international normalized ratio (INR). A routine 12-lead electrocardiogram (EKG), computerized tomography of the heart (CT), 48-Holter monitor and transthoracic echocardiogram were also obtained at that time.

\section{Pulmonary Vein Antrum Isolation Procedure}

Each patient underwent transesophageal echocardiography to exclude left atrial appendage throm- 
bus prior to the procedure. PVAI was performed using an intracardiac echocardiography (ICE) guided technique, which has been summarized here but detailed elsewhere. ${ }^{10}$ After femoral venous access was obtained, a phased-array ultrasound imaging catheter (Siemens AG, Malvern, PA, USA) was positioned in the right atrium. Under ICE guidance, a decapolar circular mapping catheter and 8-mm tip ablation catheter (Biosense Webster Inc., Diamond Bar, CA, USA) were advanced into the left atrium (LA) via two transseptal punctures. Systemic anticoagulation was achieved with the administration of intravenous heparin to maintain an activated clotting time of 350 to 400 seconds. ICE was then used to define the pulmonary venous antra and guide sequential placement of the circular mapping catheter in all positions surrounding (and outside of) each pulmonary vein (PV) radiofrequency ablation was performed wherever PV potentials were recorded surrounding the PV antra. The radiofrequency energy output was limited to a maximum of $70 \mathrm{~W}$ and $55^{\circ} \mathrm{C}$ and was titrated according to the microbubble formation detected by ICE. The end point of ablation was complete electrical disconnection of the PV antra from the LA. This was considered achieved when no PV potentials could be recorded along the antrum or inside the vein by the circular mapping catheter during sinus rhythm or with coronary sinus pacing. At the end of the procedure, all four pulmonary vein antra were remapped with the circular mapping catheter to check for any persisting or recovered PV potentials; and if necessary, further ablation was performed to eliminate these. All four PV were isolated in the $\mathrm{L}$ and the $\mathrm{R}$ atria. The superior vena cava was also isolated if high output pacing along the high lateral right atrium did not stimulate the phrenic nerve. Ablation lines between the mitral annulus and the PV were also made.

Antiarrhythmic medications were discontinued after 2 months. The antiarrhythmic medications could be restarted at the discretion of the treating physician after 2 months. All reinitiations of antiarrhythmic medications were noted. After 3 and 6 months, warfarin was continued at the discretion of the treating physician. All patients were monitored for pulmonary-vein stenosis at 3 months and, if a second procedure was performed, at 6 months. A second pulmonary-vein isolation procedure or a procedure for atrial flutter could be performed at 3 months if atrial fibrillation or atrial flutter recurred. If patients underwent a second ablation, they were followed for a total of 6 months after the last ablation, and the 6-month analysis was done using these follow-up data.

\section{Anticoagulant Therapy}

All patients were loaded with warfarin following the procedure with the goal of maintaining an international normalized ratio of 2.0-3.0. Anticoagulation was discontinued if sinus rhythm was maintained for 6 weeks without any episodes of symptomatic or asymptomatic $\mathrm{AF}$ and in the absence of other clinical indications.

\section{Follow-up}

Patients were seen at 3, 6, and 12 months after the procedure and yearly after this. Follow-up was performed with the use of a loop event monitor that patients wore at months 2 through 6 after the procedure. Patients were instructed to record any symptoms and to record at least two or three transmissions weekly even if they were asymptomatic. At each subsequent visit, EKG, Holter monitoring and weekly transtelephonic arrhythmia monitoring, and transthoracic echocardiograms were performed. Pacemakers, if present, were interrogated to look for atrial arrhythmias or mode switches. At the 3-month visit, a repeat computerized tomography of the heart was obtained to evaluate for pulmonary-vein stenosis and CRP and BNP were measured. All PVAI patients were instructed to discontinue antiarrhythmic drug therapy at 2 months after Holter monitoring.

\section{End Points}

Success was defined as freedom from AF starting two months after PVAI in the absence antiarrhythmic therapy (AAT) until the end of follow-up (defined as the last follow-up up visit from the date of PVAI). Any evidence of atrial fibrillation (symptomatic or asymptomatic) lasting more than 30 seconds during Holter or event monitoring occurring more than 2 months following PVAI or the need for redo PVAI was considered a failure. Partial success was defined as freedom from symptomatic $\mathrm{AF}$ at least 2 months after PVAI in the presence of antiarrhythmic therapy. Combined success was defined as the combination of success and partial success. The initial 2-month period was used to account for AF recurrences and hospitalizations that occur during antiarrhythmic drug titration and by the accepted belief that AF recurrence early after PVAI may be transient and therefore not representative an atrial arrhythmia recurrence. 
Table 1. Patient Characteristics

\begin{tabular}{|c|c|c|c|c|c|}
\hline Characteristic & $\begin{array}{l}\frac{\mathrm{CHD}^{*}}{\mathrm{~N}=36} \\
\end{array}$ & $\begin{array}{l}\mathrm{NSHD}^{*} \\
\mathrm{~N}=355\end{array}$ & $\begin{array}{l}\text { NSHD } \\
\text { Propensity- } \\
\text { Matched } \\
\mathrm{N}=36\end{array}$ & $\begin{array}{l}P \text { value } \\
(1) \dagger\end{array}$ & $\begin{array}{l}P \text { value } \\
(2) \ddagger\end{array}$ \\
\hline Age (years) & $53 \pm 2$ & $57 \pm 2$ & $52 \pm 2$ & $<.001$ & .82 \\
\hline Gender ( M/F) & $24: 12$ & 259:96 & 22:14 & .33 & .62 \\
\hline AF type $(n)$ & & & & .23 & .88 \\
\hline Paroxysmal & 26 & 188 & 24 & & \\
\hline Persistent & 10 & 140 & 10 & & \\
\hline Permanent & 4 & 27 & 6 & & \\
\hline Duration of AF (years) & $6 \pm 4$ & $6 \pm 4$ & $6 \pm 4$ & 1 & 1 \\
\hline Diabetes $(\mathrm{n})$ & 2 & 133 & 3 & $<.001$ & .64 \\
\hline Hyperlipidemia (n) & 10 & 190 & 7 & $<.001$ & .40 \\
\hline Hypertension (n) & 11 & 27 & 14 & $<.001$ & .45 \\
\hline LVEF (\%) & $48.6 \pm 1.32$ & $50.9 \pm 1.03$ & $52 \pm 2$ & .23 & .08 \\
\hline $\mathrm{LA}(\mathrm{cm})$ & $4.5 \pm 0.05$ & $5.9 \pm 0.02$ & $4.4 \pm 0.1$ & .23 & .97 \\
\hline BNP (ng/dL) & $161 \pm 12$ & $114 \pm 16$ & $72 \pm 6$ & .08 & .005 \\
\hline Ultrasensitive CRP (ng/mL) & $2.5 \pm 0.4$ & $4.2 \pm 5.4$ & $3 \pm 0.2$ & .24 & .65 \\
\hline No. of previously ineffective antiarrhythmic drugs & $4 \pm 1$ & $4 \pm 1$ & $4 \pm 1$ & 1 & 1 \\
\hline
\end{tabular}

${ }^{*}$ Adults with congenital heart disease (CHD) and noncongenital structural heart disease (NSHD).

$\dagger P$ value (1) between CHD and NSHD groups.

$\ddagger P$ value (2) between CHD and NSHD propensity matched groups.

\section{Statistical Analysis}

We compared long-term outcomes for those with NSHD and CHD who underwent PVAI for the treatment of drug refractory atrial fibrillation. To reduce the effects of selection bias and potential confounding in this observational study, we performed an adjustment for significant differences in the baseline characteristics of patients with the use of propensity-score matching. ${ }^{11}$ After all the propensity-score matches were performed, we compared baseline covariates between the two groups. Continuous variables were compared with the use of paired $t$-test or the chi-square testing. In the propensity-score-matched cohort, the risks of each outcome were compared with the use of Cox regression models. Survival curves were constructed with the use of Kaplan-Meier estimates and compared.

\section{Results}

\section{Study Population}

The characteristics of patients in both groups are shown in Table 1. Patients with NSHD were significantly older and had a higher incidence of hypertension, coronary artery disease, diabetes, hyperlipidemia and number of AAT used in comparison to patients with $\mathrm{CHD}$. The most common type of atrial fibrillation was paroxysmal atrial fibrillation in both groups, and there were no differences in the cumulative frequency of different types of atrial fibrillation. There were no signifi-
Table 2. Types of Congenital Heart Disease

\begin{tabular}{lc}
\hline Type of Congenital Heart Disease & $\mathrm{n}(\%)$ \\
\hline Atrial septal defect (ASD) & $22(61)$ \\
Ventricular septal defect (VSD) & $6(17)$ \\
ASD and VSD & $3(8)$ \\
Tetralogy of Fallot & $1(3)$ \\
Double outlet left ventricle and transposition of the & $1(3)$ \\
$\quad$ great vessels & $1(3)$ \\
Coarctaction of the aorta & $3(8)$ \\
Epstein anomaly & $2(6)$ \\
Bland-Garland White syndrome with cardiomyopathy & \\
\hline
\end{tabular}

cant differences in the ejection fraction or baseline preprocedural $\mathrm{BNP}$ or CRP between the two groups. The propensity-matched groups were also similar in all baseline characteristics except for BNP, which was higher in CHD group $(P<.05)$.

The individual lesions the patients with congenital heart disease are shown on Table 2. The most common congenital heart defect was atrial septal defect $(n=22,2$ closed percutaneously with an Amplatzer septal occluder (AGA Medical, Plymouth, MN, USA), 8 closed with a Dacron patch, 10 closed surgically with a pericardial patch, and the remainder were unclosed), followed by ventricular septal defects $(n=6)$, all of which were surgically repaired. The mean age was $53.4 \pm 2$ years and the median duration of follow-up was 7 months. In patients with CHD, ablation sites most commonly used were the pulmonary veins, right atrial and superior vena cava junction followed by the left atrial septum, posterior wall, coronary sinus, and crista terminalis, as shown in Figure 1. 


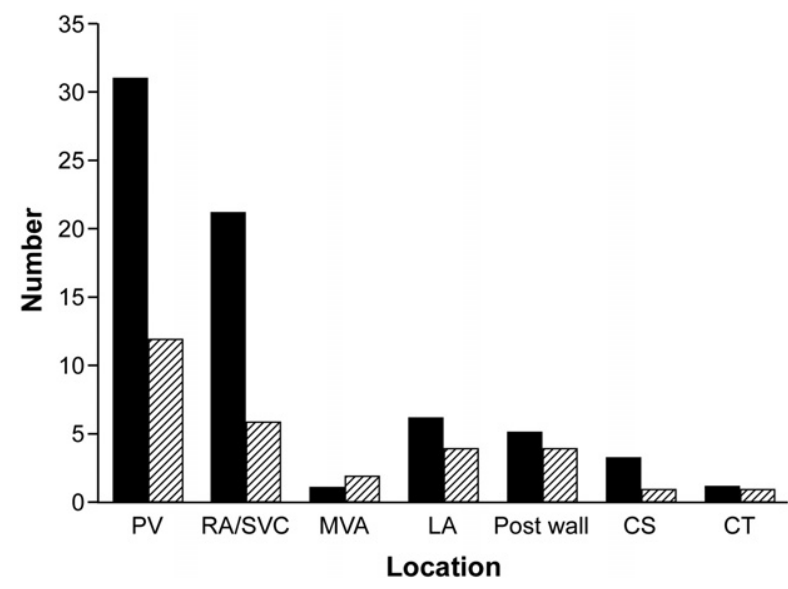

Figure 1. Ablation sites in adults with congenital heart disease. $\mathrm{PV}$, pulmonary vein; RA, right atria; SVC, superior vena cava; MVA, mitral valve annulus; LA, left atria; post wall, posterior wall; CS, coronary sinus; CT, crista terminalis.

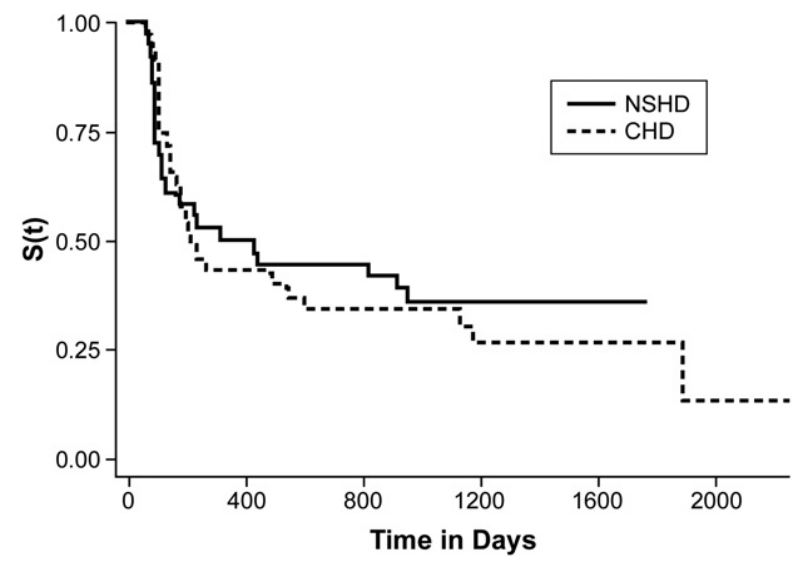

Figure 2. Kaplan-Meier survival curves for success after first pulmonary vein isolation for drug refractoryatrial fibrillation. NSHD, noncongenital structural heart disease; CHD, congenital heart disease.

\section{Clinical Outcomes}

Success rates (no symptomatic or asymptomatic atrial fibrillation in the absence of antiarrhythmic therapy) after a single PAVI in the propensitymatched CHD and NSHD groups are presented in Figure 2. After the first PVAI, success was achieved in $42 \%$ and $53 \%$ at 300 days in the CHD and NSHD groups, respectively. At the end of follow-up, at 4 years, success was $27 \%$ and $36 \%$ in the CHD and NSHD groups, respectively. There was no significant difference in the success rates between patients with CHD vs. those with NSHD $(P=.46)$.

Combined success (no symptomatic atrial fibrillation while on antiarrhythmic therapy) needing

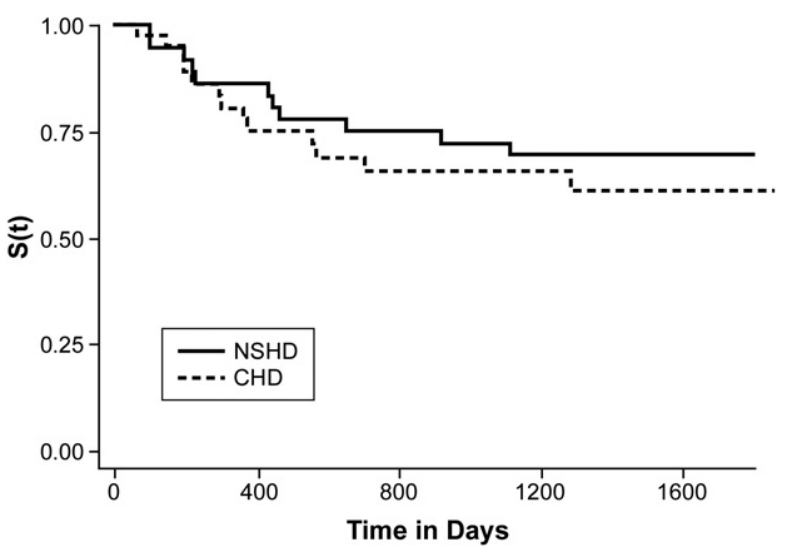

Figure 3. Kaplan-Meier survival curves for combined success after first pulmonary vein isolation for drug refractory Atrial fibrillation. NSHD, noncongenital structural heart disease; CHD, congenital heart disease.

after one PVAI in the propensity matched CHD and NSHD groups are presented in Figure 3. We found that combined success was achieved in $84 \%$ and $86 \%$ at 300 days after PVAI in the CHD and NSHD groups, respectively. At 4 years, combined success was achieved in $61 \%$ and $69 \%$ of patients in the CHD and NSHD groups, respectively. There was no significant difference in the combined success rates in patients with CHD vs. those with NSHD at the end of follow-up $(P=.69)$. The two patients in the CHD group with tetralogy of Fallot and transposition of the great vessels had difficult ablations as many of the ablation catheters were not designed to reach the left atrium and pulmonary veins and they both failed ablation attempts. There were also no significant differences in the left atrial size or changes in ejection fraction after one or two PVAI in either population of patients (Figure 4A, B)

There was no statistical in the mean complication rate between the CHD and NSHD groups $(17 \%$ vs. $11 \%, P=.42)$ as shown Table 3 . The most common complications seen were vascular access site problems followed by pulmonary vein stenosis, which occurred in two patients. Vascular access site complications were more common in patients with CHD when compared to patients with NSHD ( $8 \%$ vs. $1 \%, P<.05$ and were mostly hematomas $(90 \%)$.

\section{Discussion}

To our knowledge this is the first study to specifically examine the success of PVAI as a treatment modality for drug refractory atrial fibrillation in 
A

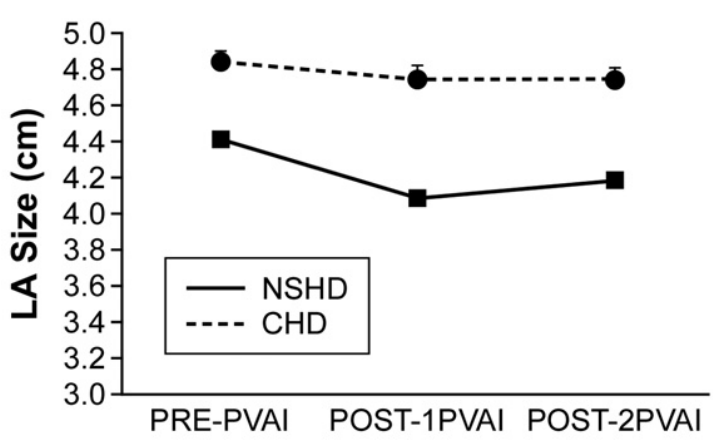

B

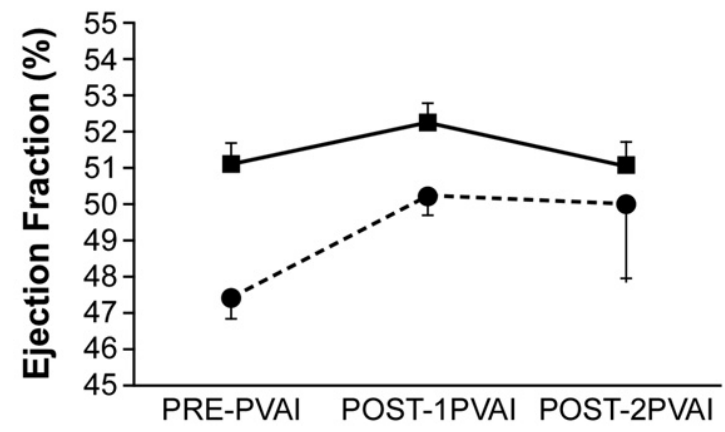

Figure 4. Changes in left atrial size (A) and ejection fraction (B) after PVAI.

Table 3. Complications by Patient Group

\begin{tabular}{llccc}
\hline & \multicolumn{1}{c}{$\mathrm{CHD}$} & & $\mathrm{NSHD}$ & \\
Complications n (\%) & $\mathrm{n}=36$ & & $\mathrm{n}=355$ & \\
\hline Mean & $6(17 \%)$ & & $38(11 \%)$ & .42 \\
Vascular access site & $3(8 \%)$ & & $5(1 \%)$ & $<.05$ \\
Embolic event & $1(3 \%)$ & & $0(0 \%)$ & .5 \\
Pulmonary stenosis & $2(5 \%)$ & $29(8 \%)$ & .47 \\
Stroke & $0(0 \%)$ & & $4(1 \%)$ & .5 \\
\hline
\end{tabular}

Adults with congenital heart disease (CHD) and noncongenital structural heart disease (NSHD).

adults with CHD. We compared outcomes in patients with CHD to patients with NSHD. We used a robust definition of success and attempted to detect the largest possible number of AF recurrences, both asymptomatic and symptomatic, with the use of Holter monitoring, ECG recordings, and transtelephonic recording of ECG. We also assessed the success of an ablative approach and success rates of ablation augmented with antiarrhythmic therapy. Patients with NSHD were older and had a higher incidence of hypertension, diabetes, and hyperlipidemia. The two groups were similar in terms of left ventricular ejection fraction, duration of atrial fibrillation and number of antiarrhythmic drugs used. We corrected for selection bias and potential confounding in this study through the use of propensity-score matching, a well-established technique; the baseline characteristics were then appropriately similar in both groups to allow for an appropriate comparison of outcomes. In the propensity-matched groups, there were no statistically significant differences between the success rate after the first PVAI in patients with CHD and patients with NSHD at any time point from time of PVAI until the end of follow-up at 4 years $(27 \%$ vs. $36 \%, P=$ .46). There were also no statistically significant differences in the combined success rates after one PVAI in patients with CHD vs. NSHD at the end of follow-up (69\% vs. $61 \%, P=.69)$. The factors that have previously been shown to influence success rate after PVAI include the type of AF, hypertension, ${ }^{12}$ left atrial dimension $(<4.5 \mathrm{~cm})$, presence of left atrial scar, and the clinical characteristics of the study population. Published early experiences after $\mathrm{AF}$ ablation mainly involved patients with paroxysmal AF and a low incidence of structural heart disease, ${ }^{13,14}$ whereas later studies have included patients with persistent $\mathrm{AF}$ (up to $38 \%$ ) and a higher incidence of structural heart disease (up to $63 \%$ ). ${ }^{15}$ Success rates similar to ours after one year of follow-up were noted by Stabile et al. in a population of patients with a high incidence of structural heart lesions (63\%). ${ }^{16}$ Our patients all had structural heart disease (congenital and noncongenital), had a high incidence of paroxysmal atrial fibrillation (66\% and $72 \%)$, and most had an enlarged left atrial dimension $(>4.5 \mathrm{~cm})$. All these characteristics influence the overall success rate and help to explain why it appears lower than previously reported success rates. ${ }^{16-20}$

$\mathrm{AF}$ originates from the interaction of a trigger and a substrate. Although the trigger plays the major role in paroxysmal AF, as left atrial remodeling develops, atrial substrate becomes more important. The maintenance of atrial fibrillation will depend greatly on the whether the atrial substrate has the capacity for reverse remodeling and if ablation can facilitate this. There is evidence to suggest that RFA applications around PV ostia during mitral valve surgery results in the restoration of sinus rhythm in $80 \%$ of patients with chronic $\mathrm{AF}$ and dilated atria, while other patients with a mean LA diameter of $60 \mathrm{~mm}$ and chronic $\mathrm{AF}$ experience a success rate of only $60 \% .^{21}$ The degree of atrial reverse remodeling may be affect success at maintenance of sinus rhythm. Patients with CHD may have had significant, long standing 
structural and electrophysiologic remodeling which inevitably effects procedural success. We witnessed no significant changes in left atrial dimensions and no significant change in ejection fraction in patients with CHD after PVAI. The lack of left atrial remodeling noted in our patients may represent the presence of significant left atrial scar, which has been reported as a powerful, independent predictor of PVAI failure. There are also procedural challenges with PVAI ablation in patients with CHD including complex anatomical considerations that do not allow typical catheter placement or impede trans-septal puncture and the ability to adequately modify the underlying electrical substrate. Pratola et al. noted persistent PV reconnection in $62.5 \%$ of patients who had normal LA size and PVAI who were followed up for 2.5 years. ${ }^{21}$ These data do suggest, however, that PVAI may sufficiently reduce the burden of AF, and together with AAT, may serve as a "hybrid" therapy to significantly control this arrhythmia in this complex patient population. A recent survey on the efficacy and safety of catheter ablation was published in 2005 involving 6000 patients and showed an average cure rate of $52 \%$ (freedom from symptomatic $\mathrm{AF}$ in the absence of antiarrhythmic therapy) and a major complication rate of $6 \% .{ }^{22}$ The main limitation of AF ablation remains the high incidence of major complications, particularly when compared to those reported during ablation of other supraventricular arrhythmias.

In patients with CHD and NSHD we found that the mean total complication rate was slightly higher than previously published $17 \%$ (CHD) and $11 \%$ (NSHD) vs. $6 \%$, although there were no statistical differences in mean complication rates between the two groups. There were more vascular access site complications in patients with CHD in comparison to those with NSHD. Awareness of this fact and application of careful preprocedural preparation may help operators reducing this complication in the future.

\section{Limitations}

This is a small retrospective analysis, although clinical data was prospectively collected. The overall ejection fraction was preserved and may limit our ability to apply these data to patients with more severe ventricular dysfunction.

\section{Conclusions}

PVAI appears to be an attractive treatment modality in drug refractory AF in adults with congenital heart disease, with success rates of $42 \%$ at 300 days and $27 \%$ at 4 years and a combined success rate in excess of $60 \%$. The success rates after PVAI in adults with congenital heart disease was similar to those with other structural heart lesions. These data suggest that referral to centers experienced in atrial fibrillation ablation for the treatment of drug refractory atrial fibrillation should be considered in adults with congenital heart disease.

\section{Author Contributions}

FP: Wrote the initial proposal, obtained IRB approval, did the data collection and statistical analysis and wrote the manuscript;

KM: Assisted in data collection

SA: Assisted in the statistical analysis

WS: Edited the manuscript

AN: Edited the manuscript

RAK: Edited the manuscript

Corresponding Author: Femi Philip, MD, Cleveland Clinic Foundation, 9500 Euclid Avenue, J2-4, Cleveland, $\mathrm{OH}$ 44195, USA. Tel: (+216) 445-7430; Fax: (+216) 445 6163; E-mail: philipf@ccf.org

Conflict of interest: None.

Accepted in final form: February 2, 2012.

\section{References}

1 Warnes CA, Williams RG, Bashore TM. ACC/ AHA 2008 guidelines for the management of adults with congenital heart disease: a report of the American College of Cardiology/American Heart Association Task Force on Practice Guidelines (Writing Committee to Develop Guidelines on the Management of Adults With Congenital Heart Disease). Developed in Collaboration With the American Society of Echocardiography, Heart Rhythm Society, International Society for Adult Congenital Heart Disease, Society for Cardiovascular Angiography and Interventions, and Society of Thoracic Surgeons. 7 Am Coll Cardiol. 2008;52:e1-121.

2 Go AS, Hylek EM, Phillips KA. Prevalence of diagnosed atrial fibrillation in adults: national implications for rhythm management and stroke prevention: the AnTicoagulation and Risk Factors in Atrial Fibrillation (ATRIA) Study. FAMA. 2001;285:23702375.

3 Wyse DG, Waldo AL, DiMarco JP. A comparison of rate control and rhythm control in patients with atrial fibrillation. $N$ Engl 7 Med. 2002;347:18251833. 
4 Hagens VE, Ranchor AV, Van Sonderen E. Effect of rate or rhythm control on quality of life in persistent atrial fibrillation. Results from the Rate Control Versus Electrical Cardioversion (RACE) Study. 7 Am Coll Cardiol. 2004;43:241-247.

5 Carlsson J, Miketic S, Windeler J. Randomized trial of rate-control versus rhythm-control in persistent atrial fibrillation: the Strategies of Treatment of Atrial Fibrillation (STAF) study. 7 Am Coll Cardiol. 2003;41:1690-1696.

6 Pedersen OD, Brendorp B, Elming H. Does conversion and prevention of atrial fibrillation enhance survival in patients with left ventricular dysfunction? Evidence from the Danish Investigations of Arrhythmia and Mortality ON Dofetilide/ (DIAMOND) study. Card Electrophysiol Rev. 2003; 7:220-224.

7 Deedwania PC, Singh BN, Ellenbogen K. Spontaneous conversion and maintenance of sinus rhythm by amiodarone in patients with heart failure and atrial fibrillation: observations from the veterans affairs congestive heart failure survival trial of antiarrhythmic therapy (CHF-STAT). The Department of Veterans Affairs CHF-STAT Investigators. Circulation. 1998;98:2574-2579.

8 Corley SD, Epstein AE, DiMarco JP. Relationships between sinus rhythm, treatment, and survival in the Atrial Fibrillation Follow-Up Investigation of Rhythm Management (AFFIRM) Study. Circulation. 2004;109:1509-1513.

9 Verma A, Natale A. Should atrial fibrillation ablation be considered first-line therapy for some patients? Why atrial fibrillation ablation should be considered first-line therapy for some patients. Circulation. 2005;112:1214-1222; discussion 1231.

10 Khan MN, Jais P, Cummings J. Pulmonary-vein isolation for atrial fibrillation in patients with heart failure. $N$ Engl 7 Med. 2008;359:1778-1785.

11 Blackstone EH. Comparing apples and oranges. 7 Thorac Cardiovasc Surg. 2002;123:8-15.

12 Berruezo A, Tamborero D, Mont L. Pre-procedural predictors of atrial fibrillation recurrence after circumferential pulmonary vein ablation. Eur Heart 7 . 2007;28:836-841.
13 Pappone C, Santinelli V, Manguso F. Pulmonary vein denervation enhances long-term benefit after circumferential ablation for paroxysmal atrial fibrillation. Circulation. 2004;109:327-334.

14 Tsai CF, Tai CT, Hsieh MH. Initiation of atrial fibrillation by ectopic beats originating from the superior vena cava: electrophysiological characteristics and results of radiofrequency ablation. Circulation. 2000;102:67-74.

15 Pappone C, Oreto G, Rosanio S. Atrial electroanatomic remodeling after circumferential radiofrequency pulmonary vein ablation: efficacy of an anatomic approach in a large cohort of patients with atrial fibrillation. Circulation. 2001;104:2539-2544.

16 Stabile G, Bertaglia E, Senatore G. Catheter ablation treatment in patients with drug-refractory atrial fibrillation: a prospective, multi-centre, randomized, controlled study (Catheter Ablation For The Cure Of Atrial Fibrillation Study). Eur Heart 7 . 2006;27:216-221.

17 Oral H, Scharf C, Chugh A. Catheter ablation for paroxysmal atrial fibrillation: segmental pulmonary vein ostial ablation versus left atrial ablation. Circulation. 2003;108:2355-2360.

18 Pappone C, Rosanio S. Evolution of nonpharmacological curative therapy for atrial fibrillation. Where do we stand today? Int 7 Cardiol. 2003; $88: 135-142$.

19 Pappone C, Rosanio S, Augello G. Mortality, morbidity, and quality of life after circumferential pulmonary vein ablation for atrial fibrillation: outcomes from a controlled nonrandomized longterm study. 7 Am Coll Cardiol. 2003;42:185-197.

20 Allessie M, Ausma J, Schotten U. Electrical, contractile and structural remodeling during atrial fibrillation. Cardiovasc Res. 2002;54:230-246.

21 Pratola C, Baldo E, Notarstefano P. Radiofrequency ablation of atrial fibrillation: is the persistence of all intraprocedural targets necessary for long-term maintenance of sinus rhythm? Circulation. 2008;117:136-143.

22 Cappato R, Calkins H, Chen SA. Worldwide survey on the methods, efficacy, and safety of catheter ablation for human atrial fibrillation. Circulation. 2005; 111:1100-1105. 